\title{
CHEMICAL POTENTIAL ON THE LATTICE
}

\author{
P. HASENFRATZ and F. KARSCH \\ CERN, Geneva, Switzerland
}

Received 9 March 1983

\begin{abstract}
The naive way of introducing chemical potential on the lattice leads to quadratic divergences even for free fermions. Starting from the analogy between the chemical potential and the fourth component of an abelian gauge field, a simple solution is proposed. For Wilson fermions it leads to a trivial modification of the hopping parameter of quarks propagating along the imaginary time direction.
\end{abstract}

The exciting problems of cosmology and heavy ion collisions [1] call for the introduction of finite temperature and particle density in (nonperturbative) QCD studies. There is a straightforward way of introducing a finite temperature in lattice gauge theory calculations and the lattice approach proved to be powerful in determining the critical temperature [2] and other thermodynamical quantities of pure gauge QCD $[3,4]$. There are also preliminary attempts at taking into account the effect of quarks [5].

On the other hand, the introduction of finite particle density is hindered by the problems of defining the chemical potential on the lattice in a satisfactory way. The naive generalization of the continuum prescription $[6,7]$ leads to quadratic divergences even for free fermions: in the continuum limit $(a \rightarrow 0)$ the energy density $\epsilon$ is proportional to $(\mu / a)^{2}$ instead of the correct finite result $\epsilon \sim \mu^{4}$ (for massless fermions), with $\mu$ being the chemical potential.

The naive generalization of the continuum prescription gives rise to the action

$$
\begin{aligned}
S= & a^{3} \sum_{x}\left(m a \bar{\psi}_{x} \psi_{x}+\mu a \bar{\psi}_{x} \gamma_{4} \psi_{x}\right. \\
& \left.+\frac{1}{2} \sum_{\mu=1}^{4}\left(\bar{\psi}_{x} \gamma_{\mu} \psi_{x+\hat{\mu}}-\bar{\psi}_{x+\hat{\mu}} \gamma_{\mu} \psi_{x}\right)\right),
\end{aligned}
$$

where the field $\psi$ is antiperiodic along the imaginary

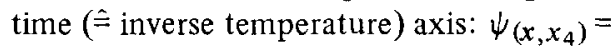
$-\psi_{\left(x, x_{4}+N_{\beta}\right)} ; N_{\beta}$ fixes the extent of the euclidean lat- tice in this direction such that $T=\beta^{-1}=\left(N_{\beta} a\right)^{-1}$ yields the temperature of the thermodynamic system.

The energy density is defined as

$\epsilon=-V^{-1} \partial \ln Z /\left.\partial \beta\right|_{\beta \mu=\text { fixed }}$,

where

$Z=\int \prod_{x} \mathrm{~d} \psi_{x} \mathrm{~d} \bar{\psi}_{x} \exp (-S)$

is the partition function of the system. After subtracting the vacuum contribution and taking the zero temperature $(\beta \rightarrow \infty)$ limit, we obtain

$$
\begin{aligned}
\epsilon= & a^{-4}\left(-\frac{1}{4 \pi^{4}}\right. \\
& \left.\times \int_{-\pi}^{\pi} \mathrm{d}^{4} q \frac{\sum_{j=1}^{3} \sin ^{2} q_{j}+(m a)^{2}}{\left(\sin q_{4}-\mathrm{i} \mu a\right)^{2}+\Sigma_{j=1}^{3} \sin ^{2} q_{j}+(m a)^{2}}\right) \\
& -a^{-4}\{\mu \equiv 0\} .
\end{aligned}
$$

It is easy to check that this expression is quadratically divergent $\left[\sim(\mu / a)^{2}\right]$ in the continuum limit. The problem is not related to the species doubling implied by eq. (1). The 16 -fold degeneracy can be removed by Wilson's prescription, but the problem discussed above, remains. Replacing the current $\bar{\psi}_{x} \gamma_{4} \psi_{x}$ by a point-split form does not help either. In order to obtain a finite result, non-covariant counterterms should be introduced in eq. (1). Although their presence is 
understandable (there is no euclidean symmetry for $\mu \neq 0$ ), it would be an awkward way to proceed.

What is the reason that no similar problems occurred in the continuum formulation? The key to understanding is the observation that in the euclidean formulation of thermodynamics the chemical potential acts like the fourth component of an imaginary, constant vector potential. In continuum QED, for instance, the chemical potential is introduced exactly as a photon field. For this reason an expansion in powers of $\mu$ is equivalent to inserting external, zero momentum photon lines to the amplitude. For instance, the contribution of order $\mu^{2}$ to the thermodynamic potential $\Omega=-(\beta V)^{-1} \ln Z$ is proportional to

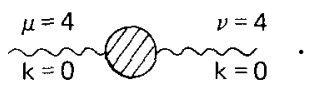

This graph is proportional to the (potentially quadratically divergent) photon mass renormalization which, however, is zero in a gauge invariant formulation ${ }^{\neq 1}$ at zero temperature (or, due to the plasmon effect, finite at finite temperature). Similarly, the finiteness of contributions of the order $\mu^{l}$ to the amplitudes $\Gamma^{(n, m)}(n$ and $m$ being the number of external photon and electron lines, respectively) follows from the renormalizability of the amplitude $\Gamma^{(n+l, m)}$. Although $l$ factors of the electromagnetic coupling constant $\mathrm{e}$ are replaced by the chemical potential $\mu$ and $l$ factors of wave function renormalization are also absent, the missing factors for charge and wave function renormalizations just cancel. This is again the consequence of gauge invariance ${ }^{\ddagger 2}$.

In eq. (1) this abelian gauge invariance is violated: $\mu$ does not enter like the fourth component of a gauge field on the lattice. The correct solution is

$$
\begin{aligned}
S= & a^{3} \sum_{x}\left(m a \bar{\psi}_{x} \psi_{x}+\frac{1}{2} \sum_{j=1}^{3}\left(\bar{\psi}_{x} \gamma_{j} \psi_{x+\hat{j}}-\bar{\psi}_{x+j} \gamma_{j} \psi_{x}\right)\right. \\
& \left.+\frac{1}{2}\left(\mathrm{e}^{\mu a} \bar{\psi}_{x} \gamma_{4} \psi_{x+\hat{4}}-\mathrm{e}^{-\mu a} \bar{\psi}_{x+\hat{4}} \gamma_{4} \psi_{x}\right)\right)
\end{aligned}
$$

$\neq 1$ On the lattice this problem is discussed in detail in ref. [8].

$\neq 2$ The importance of this generalized gauge invariance in the renormalization of theories with finite chemical potentials is emphasized by Baluni [7].
By this prescription, in eq. (4), in the integrand $\left(\sin q_{4}-\mathrm{i} \mu\right)^{2}$ is replaced by $\sin ^{2}\left(q_{4}-\mathrm{i} \mu\right)$ like in the continuum theory, and performing the $q_{4}$ integration we get

$$
\begin{aligned}
\epsilon a^{4} & =\frac{1}{2 \pi^{3}} \int_{-\pi}^{\pi} \mathrm{d}^{3} q \\
& \times \theta\left[\mathrm{e}^{\mu a}-b-\left(b^{2}+1\right)^{1 / 2}\right] b /\left(b^{2}+1\right)^{1 / 2},
\end{aligned}
$$

with

$b^{2}=\sum_{j=1}^{3} \sin ^{2} q_{j}$.

Therefore we see from eq. (6) that in every corner of the Brillouin zone the $q_{4}$ integration leads in the $a \rightarrow 0$ limit to the expected, correct result for the momentum cut-off $\sim \theta\left[\mu-\left(q^{2}+m^{2}\right)^{1 / 2}\right]$, and the resulting energy density is 16 times the usual finite energy density of free fermions at zero temperature. Using Wilson fermions, the degeneracy is removed and the factor 16 disappears for any $r \neq 0$ as it should $(0<r \leqslant 1$ is the usual arbitrary parameter in the Wilson action).

Eq. (5) can be immediately generalized to the case of QCD. At finite temperature and chemical potential the Wilson action with one flavour has the form $\neq 3$

$$
\begin{aligned}
S & =a^{3} \sum_{x}\left(\bar{\psi}_{x} \psi_{x}-K \sum_{j=1}^{3}\left[\bar{\psi}_{x}\left(\tau-\gamma_{j}\right) U_{x, x+\hat{j}} \psi_{x+\hat{j}}\right.\right. \\
& \left.+\bar{\psi}_{x+\hat{j}}\left(\tau+\gamma_{j}\right) U_{x, x+\hat{j}}^{\dagger} \psi_{x}\right] \\
& -K\left[\mathrm{e}^{\mu a} \bar{\psi}_{x}\left(\tau-\gamma_{4}\right) U_{x, x+\hat{4}} \psi_{x+\hat{4}}\right. \\
& \left.\left.+\mathrm{e}^{-\mu a} \bar{\psi}_{x+\hat{4}}\left(\tau+\gamma_{4}\right) U_{x, x+\hat{4}}^{\dagger} \psi_{x}\right]\right) \\
& +\frac{2 N}{g^{2}} \sum_{\text {plaquettes }}\left(1-N^{-1} \operatorname{Re} \operatorname{tr} U U U U\right),
\end{aligned}
$$

where the gauge fields $U$ are periodic, while the fermion fields $\bar{\psi}, \psi$ are antiperiodic along the "temperature" direction. The prescription is very simple: the hopping parameter $K$, related to the quark propaga-

\footnotetext{
${ }^{3}$ We write down the action on a symmetric lattice. For finite temperature considerations it might be convenient to introduce different lattice spacings in time and space directions. For details see ref. [3].
} 
tion by one lattice unit along the positive (negative) imaginary time axis is replaced by $\mathrm{e}^{\mu a} K\left(\mathrm{e}^{-\mu a} K\right)$.

Considering the thermodynamic potential at finite temperature, there are quark paths wrapping around the lattice in the imaginary time direction. Only these paths can lead to chemical potential dependence from ordinary closed paths the $\mu$ dependence cancels. This is understandable: ordinary loops describe virtual pair creation and annihilation, and the chemical potential of quarks and antiquarks is of opposite sign. It follows that it is not advisable to study this system at zero temperature exactly, even if we want to discuss the effect of the finite particle density alone. The hopping parameter expansion (and related iterative methods) breaks down in this case. This fact can also be seen from the explicit result for the energy density of free quarks. At zero temperature $\epsilon$ is proportional to $\theta(\mu-m(K))$, and this distribution cannot be expanded in terms of the hopping parameter. No similar problem occurs at finite temperature.

The above way of introducing a chemical potential into the lattice action of QCD leads to a quite natural extension of the calculational scheme for thermodynamic quantities in terms of a hopping parameter expansion.

We thank C. Bernard, R.V. Gavai, B. Lautrup, H. Satz and I.O. Stamatescu for discussions.
References

[1] For recent reviews, see: Q. Shafi, in: Proc. Intern. Conf. on High energy physics (Lisbon, 1981) ed. J. Dias de Deus;

L. Van Hove, in: Quark matter formation and heavy ion collisions, eds. M. Jacob and H. Satz (Bielefeld, 1982).

[2] L.D. McLerran and B. Svetitsky, Phys. Lett. 98B (1981) 195 ;

J. Kuti, J. Polonyi and K. Szlachanyi, Phys. Lett. 98B (1981) 199;

J. Engels, F. Karsch, I. Montvay and H. Satz, Phys. Lett. 101B (1981) 89;

K. Kajantie, C. Montonen and E. Pietarinen, Z. Phys. C9 (1981) 253.

[3] J. Engels, F. Karsch, I. Montvay and H. Satz, Nucl. Phys. B205 [FS5] (1982) 545.

[4] A. Billoire, G. Lazarides and Q. Shafi, Phys. Lett. 103B (1981) 450;

C.B. Lang and C. Rebbi, Phys. Lett. 115B (1982) 137;

I. Montvay and E. Pietarinen, Phys. Lett. 115B (1982) 151.

[5] J. Kuti and J. Polonyi, in: Proc. Johns Hopkins Workshop on Current problems in particle theory 6 (Florence, 1982);

J. Kogut et al., Phys. Rev. Lett. 48 (1982) 1140;

J. Kogut et al., Phys. Rev. Lett. 50 (1983) 393;

J. Engels, F. Karsch and H. Satz, Phys. Lett. 113B (1982) 398 ;

$J$. Engels and F. Karsch, The deconfinement transition for quenched SU(2) lattice in QCD with Wilson fermions, CERN preprint TH. 3481.

[6] M.B. Kislinger and P.D. Morley, Phys. Rev. D13 (1976) 2771

B.A. Freedman and L.D. McLerran, Phys. Rev. D16 (1977) 1130, 1147, 1169;

J.I. Kapusta, Nucl. Phys. B148 (1979) 461.

[7] V. Baluni, Phys. Rev. D17 (1978) 2092.

[8] B.E. Baqquie, Phys. Rev. D16 (1977) 2612. 